\title{
The Spatial Correlation of a Multiple-Input Multiple-Output and Channel Model using Huygens-Fresnel Principle for Underwater Acoustic
}

\author{
S. Kiehbadroudinezhad, A. Shahabi, and M. A. Kiehbadroudinezhad
}

\begin{abstract}
In this work, the spatial correlation of a multipleinput multiple-output (MIMO) for underwater acoustic (UWA) channel is modeled. To obtain the spatial correlation for such a channel, a mathematical method to model the effect of the surface on the acoustic propagation is studied. The sea surface has a significant impact on the underwater acoustic propagation $(U W A)$ channel since the sound field is scattered, particularly in rough sea conditions. In a situation where the sea surface is calm, the reflection is specular. In contrast, a sea surface subject to high sea states generates scattered waves. In these conditions, more complex mathematical equations are required to model the propagation. Current analytical models have limitations in terms of complexity and are not practical. Therefore, this study initially aims to consider a specular reflection to model the time-varying sea surface on the $U W A$ channel. It is a simple model with low computationally complexity and can be used to assess the performance of $U W A$ communications. Specifically, the specular reflection and transmission of an acoustic wave at a calm sea surface are studied, using the Huygens-Fresnel principle and the superposition theorem. The analytical model is developed using physical oceanic parameters representing the sea conditions. The results show a good agreement with the experimental analysis. Then, we aim to find a solution to analyze and model the surface scattering generated by the sea surface as a randomly rough surface generated by wind. The power spectrum as the Fourier transform of the correlation function of the wave height follows the Pierson and Moskowitz power spectrum which has a wide range of roughness scales. The results show a good agreement with the experimental analysis.
\end{abstract}

Index Terms-Channel Modelling, Correlation, Multiple-input multiple-output, Underwater acoustic.

\section{INTRODUCTION}

$\mathrm{T}$ he performance of a multiple-input multiple-output $(M I M O)$ depends severely on the availability of

Manuscript received August 6, 2019; revised November 20, 2019. Date of publication December 6, 2019. Date of current version December 6, 2019. The associate editor prof. Zoran Blažević has been coordinating the review of this manuscript and approved it for publication.

S. Kiehbadroudinezhad and A. Shahabi are with the Department of Electrical and Computer Engineering, Dalhousie University, Halifax, Canada (e-mails \{shahideh.k, adib.shahabi\}@dal.ca). M. A. Kiehbadroudinezhad is with the Department of Mechanic and Biosystems, University of Tehran, Tehran, Iran (e-mail: alibadrodi@alumni.ut.ac.ir).

Digital Object Identifier (DOI): 10.24138/jcomss.v15i4.888 independent multiple channels or simply, the level of correlation of the multiple channels. In perfectly uncorrelated channels between $N_{T}$ transmit sources, and $N_{R}$ receiver elements, theoretically, it is possible to multiply the capacity of the single-input single-output (SISO) channel by a factor equal to $\min \left(N_{T}, N_{R}\right)$. Since the correlated channel will decrease the performance of a MIMO system, and consequently, it's capacity, the $M I M O$ capacity mainly deepens on the correlation between the channels in addition to the number of receivers and transmitters [1-2].

To predict the spatial correlation for underwater acoustic $(U W A)$ communications, an accurate and practical acoustic channel model is necessary. The channel model is also used to predict the communication performance in realistic conditions. The model should be able to represent various conditions, as the environmental conditions of the channel and geometry have an important impact on the channel conditions. An appropriate model must enable the development of novel signal processing techniques and provide an accurate prediction of the channel to mitigate channel impairments, at low computational complexity.

To model the channel, studying the propagation of underwater sound propagation is necessary. The underwater sound intensity $\psi$ produced in an inhomogeneous medium can be developed to solve the wave equation defined as:

$$
\nabla^{2} \psi-c^{-2} \frac{\partial^{2} \psi}{\partial t^{2}}=f(r, t)
$$

where $f(r, t)$ represents the volume injection as a function of space $r$ and time $t$. Also, the speed of sound varies with depth, particularly in deep-sea deployments. There are several numerical and analytical methods to solve the above equation as described in [3]. Of particular importance are the various assumptions and approximations made in order to solve realistic propagation problems with the computational resources available. It should be considered that the Helmholtz equation is easier to solve than (1) due to the reduction in the dimension. 
It is defined as:

$$
\left[\nabla^{2}+k^{2}(r)\right] \psi(r, \omega)=f(r, \omega)
$$

Helmholtz equation is a higher-order pseudo-partial differential equation. Using numerical methods, it is a challenge to get a unique and precise solution for (2). Various techniques have been proposed. It is particularly difficult to solve the 3-D acoustic wave propagation models in shallow water, because of the multiple boundaries, and because of the different environmental parameters, such as the bottom composition, and the variable sound speed profile. Because of these nonidealities, solving the Helmholtz equation requires significant computation resources. As such, there is no universal solution technique available. The preferred technique depends on different factors [3], including dimensionality of the problem, the medium wavenumber $k(r)$, the boundary conditions, the source-receiver placement, as well as the frequency and the bandwidth of the signal of interest. To model the channel, some of the more popular methods to date are ray tracing, wavenumber integration, normal modes, parabolic equations, as well as finite difference and finite elements.

There are different techniques that can be utilized for the computation of wave fields in $2-D$ inhomogeneous media; these include the Gaussian beam approach $(G B A)$, the finite element method (FEM), the parabolic equation $(P E)$, parabolic equation solution of seismo-acoustics problems, and ocean acoustic wave propagation and ray method [4-11].

The GBA can be used in various regions with moderate computations. Whereas the Gaussian beams are frequencydependent, it can be simplified considering certain frequencydependent phenomena in the wave field such as absorption, and reflection coefficients.

Alternatively, the FEM is appropriate in a wide-angle, bottom-interacting situation environments. This method has been a reliable method for solving complex physical environments. In the case of discontinuity of geometrical shapes, the FEM has shown good performance. In this technique, the boundary conditions have been considered.

The $P E$ method for water-wave is derived based on the splitting technique of Corones. Wave propagation over a circular is considered. The $P E$ method might be applied to short wave propagation in large coastal areas of complex bottom topography. Moreover, the inhomogeneities in the ocean's sound speed profile cause refraction in long-range acoustic wave fields propagating and ray methods are sensitive to infinitely fine features.

The aforementioned methods are challenging and require significant computational resources and analysis. To validate the performance of the communication system, a lowcomplexity and flexible numerical model is required that can also represent the time-varying effects on underwater acoustic $(U W A)$ channels. To provide the analytical channel models, one needs to design a model to predict the channel behavior that can closely match the characteristics of collected data statistics. Using artificial data in a simulator enables the future development of applications testing under different conditions, and at low cost. For example, from a better understanding of the channel, estimation of the channel traffic conditions in real-time is simplified.

An important requirement for a channel model to be useful in the implementation of the communication system is that it should represent various conditions, and a statistical approach is preferred. When displacement occurs within an established system, the uncertainty known as random variations affects the channel parameters such as gain, amplitude, phase and delay variations can be represented using random variations [12].

Although the renown ray-tracing Bellhop simulator provides an approximation of the UWA channel for a given channel parameters, it does not consider random motion. The importance of a channel model has led people to develop statistical channel models and to introduce new algorithms, but they generally require excessive computation. In contrast, the main drawback of stochastic models is that they only consider the channel characteristics in a specific location, and model mainly large-scale phenomena [13].

A realistic model that can be used to evaluate the communication performance due to spatial correlation should also consider the environmental conditions such as waves at the surface, temperature, formation of bubbles near the water surface for different wind speeds, as well as seasonal effects. A combination of statistical and stochastic processes to represent the large and small scales variations can provide accurate agreement with results obtained experimentally. The statistical model can then be imported into the Bellhop beam tracer to generate a more realistic representation of the corresponding acoustic field.

The sea surface is of great interest in this work due to temporal and spatial roughness; In this work, the focus is on the evaluation of the sea surface on a propagation path. To model a precise channel all aforementioned factors must be considered separately and then integrated to obtain a robust model. It can be done as a future work.

In this study, a model is investigated for the UWA to assess the spatial correlation of a MIMO channel. The model is designed to support the prediction of the communication link's reliability prior to deployment. To validate the proposed model, the experimental data measured is used. Since the sea surface roughness varies randomly, it generates scattered waves, and to model this behavior an analytical model is developed. Initially, a specular reflection is considered to model the UWA channel to achieve a simple output that can be used for $U W A$ communication. Thus, the specular reflection and transmission of acoustic waves when the sea surface is calm is studied. Finally, the concepts are validated by comparing channel measurements to the output of the proposed model.

The remainder of this work is structured as follows. In Section II, the sea surface as a perfectly reflecting surface is studied. In Section III, spatial correlation is explained. In Section IV, the random sea surface is considered. Finally, a conclusion is drawn in Section V.

\section{The Sea Surface as a Perfectly Reflecting Surface}

The problem of the sound scattering at the sea surface due to the wind blowing has been of great interest in UWA. In real- 
world long-range propagation, the effect of the sea surface is essential due to the strong scattering of the surface roughness [14]. Therefore, we aim to find a solution to analyze and model the surface scattering generated by the sea surface in an oceanic environment.

The sea surface boundary separates the air and water, and this causes it to be considered as a perfectly reflecting surface due to the large impedance value of the water in comparison to that of air. Thus, if the sea surface is calm, the incident wave is reflected coherently with a phase shift of $180^{\circ}$ (specular reflection). The rough sea surface complicates the situation. In fact, it has been demonstrated that the scattered wave angle spread increases with increasing sea heights and in such conditions, the non-coherent addition of the multiple path arrivals creates a fading channel amplitude that varies following a Rayleigh process. In other words, the more scattered waves spread over a wider angle and consequently there will not be any specular reflection [3].

Even though surface scattering generated by waves has been considered, many of them are theoretical and complicated and not useful for actual simulation of the communication performance. Therefore, we aim to encounter this problem and find a practical solution within which the complex mathematical formulas and models are suppressed in favor of dynamical sea surface presentations.

\section{A. Two-Dimensional Model}

To calculate the field at the receiver from a perfectly reflecting surface, the Huygens-Fresnel principle and superposition theorem can be applied. The Huygens-Fresnel principle states that every point on a wave-front may be considered a source of secondary spherical wavelets that spread out in the forward direction (with the same frequency as that of the primary wave). The new wave-front is the tangential surface to all these secondary wavelets. The amplitude of the acoustic field at any point beyond is the superposition of all these wavelets with the consideration of their amplitudes and relative phases [15-16].

According to the Huygens-Fresnel principle, when a wave gets reflected from the sea surface, it always spread to some extents to regions that were not directly hit by the transmitter waves [15-16].

The Huygens-Fresnel principle is applied to derive an equation at the receiver from the surface in a region of $n$ points at the surface where the receiver is not directly exposed to the transmitted wave. Of particular importance are the various assumptions and approximations made in order to approximate the field at the receiver after getting reflected at the sea surface. We assume that in the region of $n$ points at the surface, the number of points is adequately large. Moreover, the distance between the source and the receiver is sufficiently far that the wave-fronts are approximated as planar waves with uniform amplitude from any point within the bounded region and approximate equal angles formed at the surface, and the element width of each area is much less than the wavelength [15-16].

Figure (1) illustrates an example of a reflected wave at the surface [3]. Assume that a point source located at a point $P_{0}$, vibrating at a frequency $f$. The disturbance is defined by a complex variable $U_{0}$ known as the complex amplitude. It produces a wave with wavelength $\lambda$ and wavenumber $k=2 \pi / \lambda$. Using Huygens' theory and the principle of superposition of waves, the complex amplitude at a further point $P$ is found by summing the contributions from each point on the region of length $2 l$. Only a few element widths of the region are shown in the figure. We aim to approximate the field at point $P$ for a net contribution of all $n$ points [15-16].

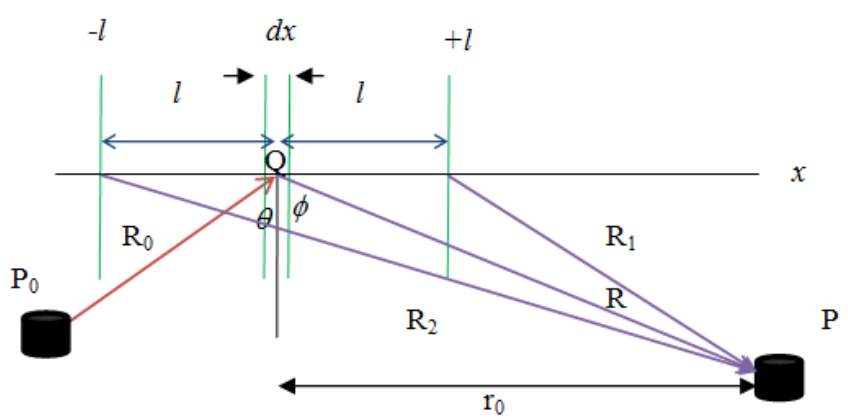

Fig. 1. Geometric arrangement for complex field calculation in 2-D.

The complex amplitude of the primary wave at the point $Q$ located at a distance $R_{0}$ from $P_{0}$ is given by:

$$
U\left(R_{0}\right)=\frac{U_{0} e^{j\left(w t-k R_{0}\right)}}{R_{0}}=U_{Q}
$$

The range $2 l$ at the surface is considered that varies from $-l$ to $+l$ along the $x$-axis (refer to Figure 1). Therefore, we need to resolve the total field of this range at point $P$.

We assume the intended range that we aim to calculate the field is located at distances $R_{1}$ and $R_{2}$ as shown in Figure 1. Thus:

$$
\begin{array}{r}
R \cong R_{1}-l \sin (\varnothing), \\
R \cong R_{2}+l \sin (\varnothing)
\end{array}
$$

Assuming further that the ranges appearing in the denominators of all terms can be replaced simply by the slant range for $R$ (the amplitudes decay slowly with range):

$$
\frac{1}{R} \cong \frac{1}{R_{1}} \cong \frac{1}{R_{2}}
$$

The complex field at the point $P$ will be:

$$
\begin{aligned}
& U_{P}=\int_{r_{0}-l}^{r_{0}+l} A U_{Q} \frac{e^{j k(R-x \sin (\varnothing))}}{R} d x= \\
& A U_{Q} \frac{e^{j k R}}{R} \int_{r_{0}-l}^{r_{0}+l} A U_{Q} e^{-j k x \sin (\varnothing)} d x= \\
& A U_{Q} \frac{e^{j k R}}{R} 2 l e^{-j k r_{0} \sin (\varnothing)} \frac{\sin (\beta)}{\beta}
\end{aligned}
$$

where $\beta=k l \sin (\varnothing)$ and $A$ is a parameter as a function of the obliquity factor and lambda. It should be considered that the $A$ parameter is assumed to be constant through the range of $-l$ to $l$. 


\section{B. Three-Dimensional Model}

In this section, a $3-D$ model is investigated to calculate the field at the receiver from a perfectly reflecting surface using the Huygens-Fresnel principle and superposition theorem. Referring to Figure (2), consider a sound source located at point $P_{0}$ and a receiver at point $P[3,15,17]$. Using Huygens' theory and the principle of superposition of waves, the complex amplitude at the point $P$ is found by summing the contributions from each point on the sphere of radius $R_{0}$.

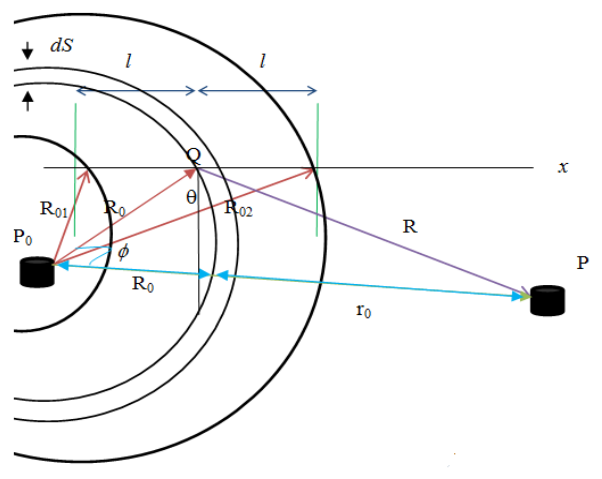

Fig. 2. Geometric arrangement for complex field calculation in 2-D.

The secondary wavelets within an infinitesimal space $d S$ located in the range of $-l$ to $l$ are coherent and they emit in phase with the primary wave.

The secondary sources travel a distance from the point $-l$ to $l$ and cause the distance $R$ where is the distance between the reflected point and the receiver, varies in the range between $R_{0}-l \sin (\theta)$ to $R_{0}-l \sin (\theta)$.

The field in the infinitesimal space resulted from the $d S$ can be calculated as:

$$
d U_{S}=U_{Q} A \frac{e^{j k R}}{R} d S
$$

where the obliquity factor $A$ is assumed to be constant through $d S$.

$$
d S=R_{0} d \emptyset 2 \pi\left(R_{0} \sin (\varnothing)\right)
$$

Using the law of cosines gives:

$$
R^{2}=R_{0}^{2}+\left(R_{0}+r_{0}\right)^{2}-2 R_{0}\left(R_{0}+r_{0}\right) \cos (\varnothing)
$$

If the distances of $R_{0}$ and $r_{0}$ remains constant, then the derivative of the above equation yields:

$$
2 R d R=2 R_{0}\left(R_{0}+r_{0}\right) \sin (\varnothing) d \emptyset
$$

Therefore:

$$
d S=2 \pi \frac{R_{0}}{R_{0}+r_{0}} R d R
$$

The total complex field at the receiver from a sphere with the radius of $R_{0}-l \sin (\theta)$ to $R_{0}+l \sin (\theta)$ is given as:

$$
\begin{gathered}
U_{P}=\int_{R_{0}-l \sin (\theta)}^{R_{0}+l \sin (\theta)} A U_{Q} 2 \pi \frac{R_{0}}{R_{0}+r_{0}} R d R \frac{e^{-j k R}}{R} \\
U_{P}=4 \pi A U_{Q} l \sin (\theta) \operatorname{sinc}(\beta)
\end{gathered}
$$

Figure 3 demonstrates the 2-D and $3-D$ of the proposed channel amplitude, where the magnitude has been normalized. The amplitude of the 3-D model is greater than that of the 2-D model. It shows that $2-D$ and $3-D$ modeling are in good agreement except for their amplitudes.

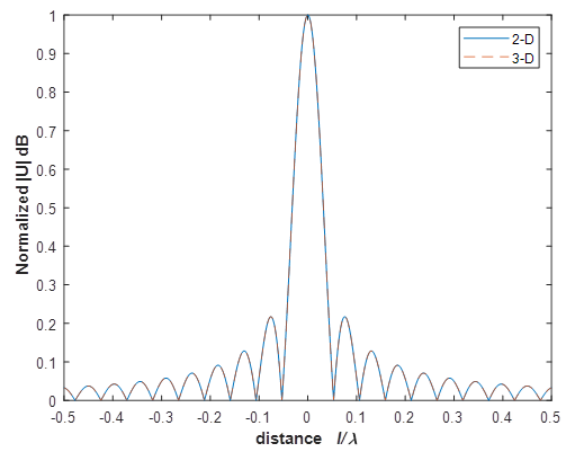

Fig. 3. Normalized field strength in $\mathrm{dB}$ versus distance in 2-D and 3-D.

\section{CORRElation OF THE CHANNEl OR SPATIAL CORRELATION}

The spatial correlation is the main channel correlation that is the correlation of the channel due to scattering [1]. After finding a proper model for the channel, we can find a solution for the spatial correlation between the channels.

The spatial correlation between the channels for a given channel matrix $H$ is as [1]:

$$
\rho_{i j, p q}=\frac{E\left\{h_{i j} h_{p q}{ }^{*}\right\}}{\sqrt{E\left\{h_{i j} h_{i j}{ }^{*}\right\} E\left\{h_{p q} h_{p q}{ }^{*}\right\}}}\left\{\begin{array}{l}
i, p=1,2, \ldots, N \\
j, q=1,2, \ldots, M
\end{array}\right.
$$

where $N$ and $M$ are the number of receivers and transmitters, respectively.

Let's assume that all the antennas are the same with Omnidirectional radiation patterns and Angle Of Arrival (AOA) at the receiver is $360^{\circ}$ perpendicular to the antennas.

For a $M I M O$ system of $U W A$, the output intensity field at the receivers $U_{s_{1}}, U_{s_{2}}, \ldots, U_{s_{N}}$ can be expressed in terms of the channels as:

$$
\left\{\begin{array}{c}
U_{s_{1}}=h_{11} U_{t}+h_{21} U_{t}+\cdots+h_{M 1} U_{t} \\
U_{S_{2}}=h_{12} U_{t}+h_{22} U_{t}+\cdots+h_{M 2} U_{t} \\
\cdot \\
\cdot \\
\cdot \\
U_{s_{N}}=h_{1 N} U_{t}+h_{2 N} U_{t}+\cdots+h_{M N} U_{t}
\end{array}\right.
$$

To simplify, we consider a MIMO system with two numbers of receivers and two transmitters. The spatial correlation of the channel of $h_{11}$ and $h_{12}$ will be calculated as follows:

$$
h_{11}=\frac{U_{s_{1}}}{U_{1}}, h_{12}=\frac{U_{S_{2}}}{U_{1}}
$$


The general formula of the correlation coefficient $\rho_{i j, p q}$ can be computed using [1-2]:

$$
\rho_{i j, p q}=\frac{E\left\{h_{i j} h_{p q}{ }^{*}\right\}}{\sqrt{E\left\{h_{i j} h_{i j}{ }^{*}\right\} E\left\{h_{p q} h_{p q}{ }^{*}\right\}}}=\frac{E\left\{U_{s_{1}} U_{S_{2}}{ }^{*}\right\}}{\sqrt{E\left\{U_{S_{1}} U_{S_{1}}{ }^{*}\right\} E\left\{U_{S_{2}} U_{S_{2}}{ }^{*}\right\}}}
$$

while the channel matrix $H$ is:

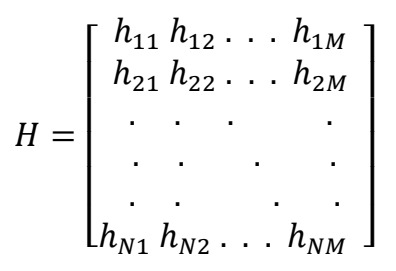

We consider the field of the first receiver as the reference point at the receivers. Thus, the field at the two receiving hydrophones $U_{t_{1}}$ and $U_{t_{2}}$ are determined in terms of the channels as:

$$
\begin{aligned}
& U_{t_{1}}=h_{11} U_{s}+h_{12} U_{s}, \\
& U_{t_{2}}=h_{21} U_{s}+h_{22} U_{s}
\end{aligned}
$$

Finally, we expand the above equations to obtain the general spatial correlation for a MIMO system as a function of wave parameters:

$$
\rho_{11,22}=\frac{E\left\{h_{11} h_{22}{ }^{*}\right\}}{\sqrt{E\left\{h_{11} h_{11}{ }^{*}\right\} E\left\{h_{22} h_{22}{ }^{*}\right\}}}=\frac{E\left\{U_{S_{1}} U_{S_{2}}{ }^{*}\right\}}{\sqrt{E\left\{U_{t_{1}} U_{t_{1}}{ }^{*}\right\} E\left\{U_{t_{2}} U_{t_{2}}{ }^{*}\right\}}}
$$

Since:

$$
\left\{\begin{array}{c}
U_{t_{1}}(\varnothing)=u_{t_{0}} \\
U_{t_{2}}(\varnothing)=u_{t_{0}} e^{j k d_{t} \cos (\varnothing)} \\
U_{s_{1}}(\varnothing)=u_{s_{0}} \\
U_{s_{2}}(\varnothing)=u_{s_{0}} e^{j k d_{s} \cos (\varnothing)}
\end{array}\right.
$$

where $U_{t_{1}}$, and $U_{t_{2}}$ are far-field amplitudes at the transmitter and $U_{s_{1}}(\varnothing)$, and $U_{s_{2}}(\varnothing)$ are far-field amplitudes at the receiver, respectively. Also, $d_{t}$ and $d_{s}$ are space elements at the transmitter and receiver, respectively.

Therefore, in a $M I M O$ system with $N$ numbers of receivers and $M$ numbers of transmitters:

$$
\rho_{i j, p q}=J_{0}\left(k d_{t}|q-j|\right) J_{0}\left(k d_{s}|p-i|\right)
$$

Figure 4 illustrates the correlation coefficient versus element space evaluated using (22). The results are implemented in MATLAB R2019a. The continuous blue curve assumes that the receiver spacing is 100 number of elements with $\lambda / 20$ interelement spacing, it is also super-imposed with 5 of elements with $\lambda / 2$ inter-element spacing.

Figure 5 illustrates the comparison between the experimental and theoretical data correlation coefficients using (22) with 5 numbers of equally spaced elements of $\lambda / 2$, respectively.

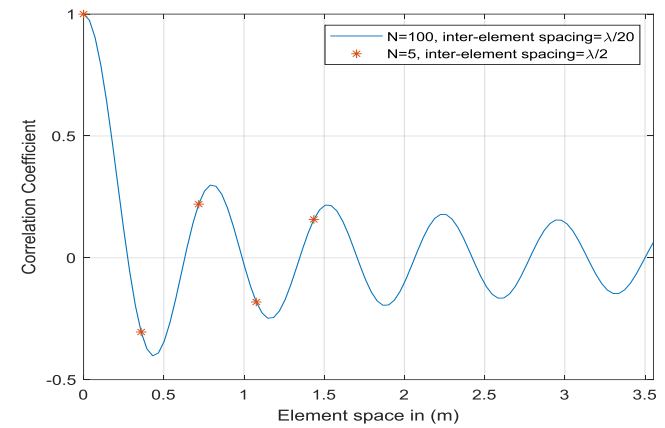

Fig. 4. Correlation coefficient versus element space for 100 number of elements with $\lambda / 20$ inter-element spacing and 5 number of elements with $\lambda / 2$ inter-element spacing.

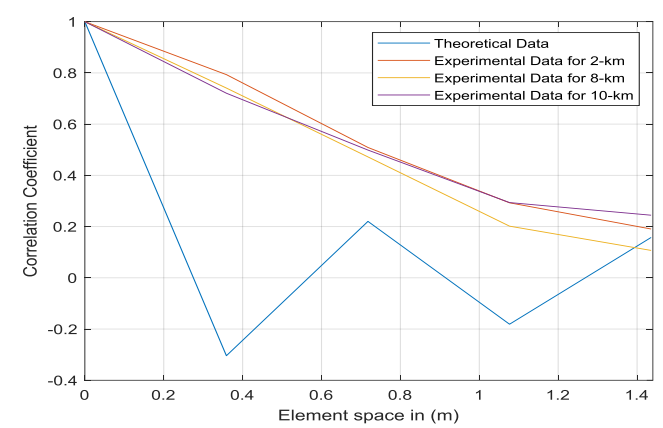

Fig. 5. Correlation coefficient versus element space for 5 number of elements experimental data for different range and theoretical data, considering a calm sea surface.

\section{THE RANDOM SEA SURFACE}

Wave motion significantly affects the underwater acoustic channel properties; however, it can be used to increase the richness of the scattered channel for underwater acoustic $(U W A)$ communication. To obtain more information about the wave surface, one needs to have knowledge about the wave height and period (or length). Depending on the sea depth, waves show different properties; height and direction of travel change with time and space. These properties are very important in coastal engineering design. Waves generated by winds blowing over the sea surface do not have the same height and period. As such, they are generated randomly, and probability/statistical models can be developed for this purpose [14].

A snapshot of the random sea demonstrates that the sea surface height has a random distribution. If we consider two points $A$ and $B$ on the sea surface, each point is represented by a collection of surfaces or ensemble of the random sea surface. Let a sample in the ensemble as a function of $r$ (distance between $A$ and $B$ ) be denoted by $\gamma^{(k)}(r)$, a function of the sample is also a random number. The most essential factors of a random process are the correlation among any numbers of points. To simplify the problem, we place an assumption of the ergodic process.

To consider a randomly rough surface generated by wind, two main parameters are the spread of heights and the change of these heights along the surface. Therefore, to obtain the variation of heights, the surface correlation function must be described. Moreover, to characterize the spread of heights, a single point height does not provide a good description. 
Therefore, we separate the random surface by their correlation functions using $S$ which is the area of the mean surface.

In this study, we assume that the power spectrum as the Fourier transform of the correlation function of the wave height follows the Pierson and Moskowitz power spectrum which has a wide range of roughness scales [18]. The objective is to describe the pressure scattering field as a function of different kinds of rough surfaces and obtain the correlation function for a $M I M O$ system. Moreover, we place an assumption that a plane wave incident on a perfectly reflecting surfaces in either calm or rough surface gets scattering, in addition to the assumption of pressure release and hard surface at these surfaces. Even though these concepts do not follow a deterministic approach, they allow us to approximate the rough sea surface.

\section{A. Sound Scattering Caused by Wave Motion at the Sea Surface}

The ocean almost encounters with rough surface which causes a complex wave motion at the surface. Turbulent wind is the source of wave motion at the sea surface and possesses a highly irregular, random character. The fundamental characteristic- the displacement of the surface resulted from the experimental observation of the wind- is a random function of the horizontal distance (space) and time. Pierson and Moskowitz demonstrated the results of the dimensional analysis of a great mass of experimental material. It was shown that the angular distribution of the wave energy is dependent on the wave frequency. A narrow angular spectrum belongs to the lowfrequency components of sea roughness and the high-frequency spectrum is isotropic [14, 19].

When a sea surface is rough, sound transmitted wave incidents the surface and exhibits scattering. The scattered sound wave consists of coherent and incoherent components. A propagated wave in the direction of specular reflection is the coherent component. The statistical averaging of the scattered field on the ensemble of the displacement function leads to the coherent component. The coherent (mean) reflection coefficient is the ratio of the amplitude of the scattered wave to that of the transmitted wave. The reflection coefficient can be close to -1 ; if the surface is even (the sea surface is perfectly reflected). But, the reflection coefficient is always practically less than unity due to the breakdown of a part of the scattered field. The coherent reflection coefficient declines with increasing the surface wave height. The experimental data illustrates that the coherence parameter has a form of Rayleigh parameter $P$ [14]:

$$
P=2 k \sigma \sin \chi
$$

where $k$ is the sound-wave number, $c$ is the grazing angle of a sound wave, and $s$ is the root-mean-square of the sea surface roughness which is $\sigma=\sqrt{\langle\gamma\rangle^{2}}$, therefore (23) can be rewritten as follows:

$$
P=2 k \sqrt{\langle\gamma\rangle^{2}} \sin \chi
$$

It is shown that for $P>>1$, the coherent component turns to zero, and the scattered field is about incoherent.

The sound scattered in the direction of the sound source which is referred as the backscattered sound is very important in underwater acoustics (UWA) communication. The backscattered sound depends on the grazing angle and at large angles greater than $60^{\circ}$, the specular reflection is responsible for the scattered field while at a smaller grazing angle, the resonant scattering is mainly of interest. At high frequencies, air bubbles play an important role in the scattering field. The effect of the air bubbles on the scattering is high when bubbles are resonant [20].

\section{B. Methods \\ B.1 Time series analysis of the wave motion}

It should be considered that generated waves on the sea surface do not form simple sinusoids. Therefore, the sea surface consists of random waves of various lengths and periods that is the sum of an infinite series of sine and cosine functions with harmonic wave frequencies (Fourier series). Determination the Fourier series of the sea surface is impractical and needs the measurements of the height of the sea surface. The description of such surface is not easy. A simple description is the approach of the spectrum of ocean waves that provides the distribution of wave energy among different wave frequencies of wave-lengths on the sea surface [14].

In order to estimate the surface wave, in this study, we assume that a point on the surface moves up and down and creates a displacement that varies sinusoidally in time with amplitude $A_{w}$ and frequency $f_{w}$. Note that only the vertical surface motion is considered [14].

To design a $U W A$ system, particularly for a dynamic system, more information on the main property of the waves, the heights, with distinct wave periods are needed. We assume a plane wave incident a rough sea surface has a finite number of Fourier components with a random phase angle over 0 and $2 p$. Considering a single sinusoidal wave that is traveling in the $x$ direction, the wave in terms of its period and height can be formulated as [14]:

$h(r, t)=A_{w} \sin \left(k_{w} r-\omega_{w} t+\phi\right)=\frac{H_{w}}{2} \sin 2 \pi\left(\frac{r}{\lambda}-\frac{t}{T_{w}}+\phi\right)$

where $A_{w}$ is the wave amplitude, $H_{w}$ is the wave height, $k_{w}$ is the wavenumber, $\omega_{w}$ is the wave frequency and $T_{w}$ is the wave period.

For a random sea summation of many individual linear waves are considered [14]:

$$
h(r, t)=\sum_{n=1}^{n=\infty} A_{w} \sin \left(k_{w} r-2 \pi f_{w n} t+\phi_{n}\right)
$$

where $A_{w}$ and $f_{w}$ are the wave amplitude (half of the wave height) and the wave frequency, respectively. With some simple modifications, (26) can be given by:

$$
\begin{gathered}
h(x, t)=\frac{H_{w}}{2} \sin \left(k_{w} x-\omega_{w} t+\phi\right), \\
H_{w}=\alpha u_{m}{ }^{2}, \\
h(x, t)=\frac{\alpha}{2} \frac{u_{m}}{c} c u_{m} \sin \left(k_{w} x-\omega_{w} t+\phi\right)= \\
\frac{\alpha}{2} a_{d w} c u_{m} \sin \left(k_{w} x-\omega_{w} t+\phi\right) .
\end{gathered}
$$

where $\mathrm{a}_{\mathrm{dw}}$ is the Doppler Effect caused by the wave motion and $\mathrm{u}_{\mathrm{m}}$ is the wave speed. 
We assume further that the ranges appearing in the denominators of all terms can be replaced simply by the slant range for the reference point (the amplitudes decay slowly with range) but cannot be negligible for the phase effect. Moreover, we assume that the transmitter, receiver, boat, sea surface, and any other movements introduce a drift in directions $\emptyset_{d f}$ with respect to horizontal plane. Therefore:

$$
\begin{gathered}
R-d \cos \left(\theta_{d}+\emptyset_{d f}\right)=R-d\left[\cos \left(\theta_{d}\right) \cos \left(\emptyset_{d f}\right)-\right. \\
\left.\sin \left(\theta_{d}\right) \sin \left(\emptyset_{d f}\right)\right]
\end{gathered}
$$

where $R, \emptyset_{d f}, \theta_{d}$, and $d$ are the reference path, total drifting angle, angle between the array elements, and distance between the elements, respectively.

\section{1) Correlation of the channel due to scattering or spatial correlation}

We mentioned that sea surface exhibits a random process; consequently, the scattered pressure field is random though which provides knowledge about the distribution of the pressure field. However, the scattered pressure field does not characterize the sea surface in terms of deterministic quantity. In contrast, the spatial correlation quantity provides information about the coherence of the random pressure.

The spatial correlation function of two points on the sea surface is:

$$
B_{s}(\bar{r}, \eta)=\left\langle p_{s}\left(r_{1}, z_{1}\right) p_{s}^{*}\left(r_{1}, z_{1}\right)\right\rangle
$$

We assume that all the antennas are the same with omnidirectional radiation patterns and $A O A$ at the receiver is $360^{\circ}$ perpendicular to the antennas.

For a $M I M O$ system of $U W A$, the output intensity field at the receivers $p_{s 1}, p_{s 2}, \ldots, p_{s N}$ can be expressed in terms of the channels as:

$$
\begin{gathered}
p_{s 1}=h_{11} p_{t}+h_{21} p_{t}+\ldots+h_{M 1} p_{t} \\
p_{s 2}=h_{12} p_{t}+h_{22} p_{t}+\ldots+h_{M 2} p_{t} \\
\cdot \\
\cdot \\
\cdot \\
p_{s N}=h_{1 N} p_{t}+h_{2 N} p_{t}+\ldots+h_{M N} p_{t}
\end{gathered}
$$

We expand the above equation to obtain the general spatial correlation for a MIMO system as a function of wave parameters, surface roughness, and array spacing elements.

Finally, in a $M I M O$ system with $N$ numbers of receivers and $M$ numbers of transmitters, the correlation coefficient is as follows:

$$
\begin{aligned}
& \rho_{i j, p q}=J_{0}\left(k d_{t} \alpha_{c o f f}|q-j| \cos \left(\emptyset_{d f}\right)\right) J_{0}\left(k d_{t} \alpha_{c o f f} \mid q-\right. \\
& \left.j \mid \sin \left(\emptyset_{d f}\right)\right) J_{0}\left(k d_{r} \alpha_{c o f f}|q-j| \cos \left(\emptyset_{d f}\right)\right) J_{0}\left(k d_{r} \alpha_{c o f f} \mid q-\right. \\
& \left.j \mid \sin \left(\emptyset_{d f}\right)\right) \quad
\end{aligned}
$$

where $\alpha_{c o f f}=\frac{\alpha}{2} a_{d w} c u_{m}$.

Figure 6 illustrates the comparison between the experimental and theoretical data correlation coefficients using the above equation with 5 numbers of equally spaced elements of $\lambda / 2$, respectively. The experimental data were taken during summer when the water was calm which led the value of $\alpha_{\text {coff }}$ to be 0.25 . Furthermore, the hydrophones were towed to a boat during the experiment and caused a big drifting due to the boat movement $\left(\emptyset_{d f}=35^{\circ}\right)$.

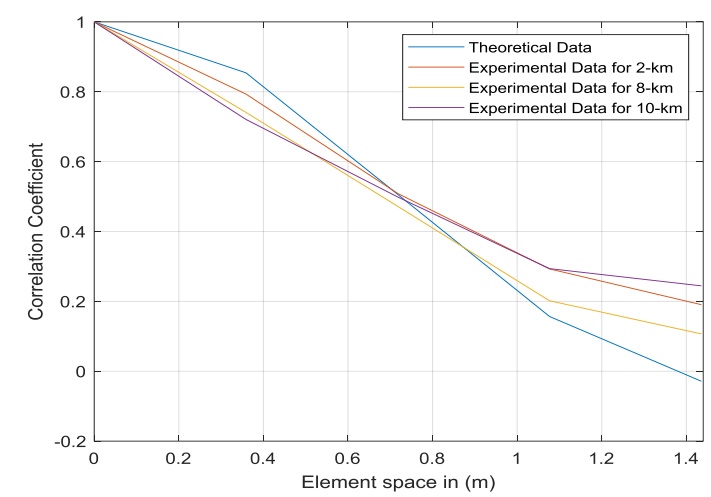

Fig. 6. Correlation coefficient versus element space for 5 number of elements experimental data for different range and theoretical data, considering a wavy sea surface.

\section{CONCLUSION}

This paper presented an analytical model for a multiple-input multiple-output (MIMO) channel correlation for underwater acoustic (UWA) communications. To predict the spatial correlation for underwater acoustic ( $U W A$ ) communications, an accurate and practical acoustic channel model is necessary. A model was developed to include the effect of the time-varying sea surface on the propagation conditions. In contrast to the existing correlation models, our new approach can predict the channel behavior precisely prior to deployments. The model developed can be applied to a ray-tracing algorithm to generate the corresponding acoustic field. In this work, we focus particularly on the effect of wind in induced waves at the surface. Therefore, we initially aim to find a solution to analyze and model the surface scattering generated by the sea surface as a perfectly reflecting surface in an oceanic environment. As such, $2-D$ and $3-D$ models were investigated to calculate the field at the receiver from a perfectly reflecting surface using the Huygens-Fresnel principle and superposition theorem. The results illustrated that the $2-D$ and $3-D$ modeling are in good agreement except for their amplitudes. To validate the model developed, the correlation function for the scatted field in a deep-sea due to a calm sea surface for a MIMO system is computed numerically. The proposed method is analytically easy to compute. To obtain a good insight into the proposed model, the correlation coefficient achieved during a sea trial near St-Margaret's Bay is compared to the proposed model. The results show a good agreement (with some degree off which could be due to not considering some factors precisely such as a random rough sea surface). Therefore, we then aim to find a solution to analyze and model the surface scattering generated by the sea surface as a randomly rough surface generated by wind. To design a $U W A$ system, particularly for a dynamic system, more information on the main property of the waves, the heights, with distinct wave periods are needed. We assumed a plane wave incident a rough sea surface has a finite number of Fourier components with a random phase angle. We used the 
approach of the spectrum of ocean waves that provides the distribution of wave energy among different wave frequencies of wave-lengths on the sea surface. As such, we assumed that the power spectrum as the Fourier transform of the correlation function of the wave height follows the Pierson and Moskowitz power spectrum which has a wide range of roughness scales. We also found the spatial correlation for a $M I M O$ and found the maximum received scatted field at the receiver while considering the wind parameters follows Pierson and Moskowitz's power spectrum. The correlation coefficient achieved during a sea trial near St-Margaret's Bay is compared to the proposed model. The results show a good agreement. However, the suggested model provides a simple and practical formula to model a $U W A$ channel.

\section{ACKNOWLEDGMENT}

The authors would like to thank Dr. Jean-Francois Bousquet for sharing his experimental data.

\section{REFERENCES}

[1] H. T. Hui, "Influence of antenna characteristics on MIMO systems with compact monopole arrays," IEEE J. Antennas and Wireless Propagation Letters, vol. 8, pp. 133-136, Feb. 2009. DOI: 10.1109/LAWP.2009. 2012446.

[2] H. T. Hui, and X. Wang, "Building antenna characteristics into MIMO channel simulation," International Journal of Electronics, vol. 97, no. 6 , pp. 703-714, Jun. 2010. DOI: 10.1080/00207211003646969.

[3] F. B. Jensen et al., "Computational ocean acoustics," in American institute of physics, Springer, New York, 2011, pp. 1-232. DOI: 10.1002/qj.49712 52515.

[4] V. Cerveny, M. M. Popov, and I. Psencik, "Computation of wave fields in inhomogeneous media-Gaussian beam approach," Geophysical Journal of the Royal Astronomical Society, vol. 70, pp. 109-128, Jul.1982. DOI: 10.1111/j.1365-246X.1982.tb06394.x.

[5] F. Rashidi, "An efficient finite element method for numerical modeling of shallow water wave propagation," International Journal of Intelligent Engineering and Systems, vol. 9, no. 3, 2016. DOI: 10.22266/ijies2016. 0930.16

[6] B. Yu et al., "A Parallel Implementation of Acoustic Propagation Mode on Cloud Platform," International Symposium on Parallel Architectures, Algorithms and Programming (PAAP), pp. 140-143 Oct. 2014. DOI: 10.1109/PAAP.2014.57.

[7] P. S. Petrov, M. Y. Trofimov, and A. D. Zakharenko, "Mode parabolic equations for the modeling of sound propagation in 3D-varying shallow water waveguides," IEEE Days on Diffraction (DD), no. 6402778 , pp. 197-202, 2012

[8] J. M. Collis et al., "Parabolic equation solution of seismo-acoustics problems involving variations in bathymetry and sediment thickness," $J$. Acoust. Soc. Am. vol. 123, no. 1, pp. 51-5, Jan. 2008. DOI: 10.1121/1. 2799932.

[9] A. C. Radder, "On the parabolic equation method for water-wave propagation," J. Fluid Mech, vol. 95, no. 1, pp. pp. 159-176, Nov. 1979. DOI: https://doi.org/10.1017/S0022112079001397.

[10] J. F. Lingevitch, and M. D. Collins, "Parabolic equations for gravity and acousto-gravity waves," J. Acoust. Soc. Am, vol. 105, no. 6, pp. 3049 3056, Feb. 1999. DOI: 10.1121/1.424634.

[11] K. C. Hegewisch, N. R. Cerruti, and S. Tomsovic, "Ocean acoustic wave propagation and ray method correspondence: Internal wave fine structure," J. Acoust. Soc. Am, vol. 117, no. 3, pp. 1582-1594Aug. 2005. DOI: $10.1121 / 1.1854842$

[12] A. Paulraj, R. Nabar, and D. Gore, "Introduction to space-time wireless communications," Cambridge University Press, UK, 2003, pp. 1-81.

[13] P. Qarabaqi, and M. Stojanovic, "Statistical characterization and computationally efficient modeling of a class of underwater acoustic communication channel," IEEE journal of oceanic engineering, vol. 38, no. 4, pp. 701 - 717 , Sep. 2013. DOI: 10.1109/joe.2013.2278787.

[14] P. Holmes, "Professional development programme: coastal infrastructure design, construction and maintenance," Imperial College, England Chapter 5, Coastal processes: waves, 2001.
[15] C. Huygens, Trait'e de la Lumiere, Leyden, 1690. Translated into English by S. P. Thompson, London, 1912, Reprinted by the University of Chicago Press.

[16] M. Born, and E. Wolf, "Principles of Optics," Cambridge University Press, 2006.

[17] B. E. Parkins, "Reflection and scattering from a time-varying rough surface- the nearly complete Lioyd's mirror effect," The Journal of the Acoustical Society of America, vol. 49, no. 5, pp. 1484-1490, 1971. DOI: 10.1121/1.1912523.

[18] W. J. Pierson, and L. Moskowitz, "A proposed spectral form for fully developed wind seas based on the similarity theory of S. A Kitaigorodskii," Journal of Geophysical Research, vol. 69, no. 24, pp. 5181-5190, Dec. 1964. DOI. 10.1029/JZ069i024p05181.

[19] L. M. Brekhovskikh, and Yu. P. Lysanov, "Fundamentals of ocean acoustics," 3rd ed., Springer, 2003. DOI: 10.1007/b97388.

[20] Y. Zhen, "Acoustic scattering by periodic arrays of air-bubbles," Acta Acustica united with Acustica, vol. 89, no. 3, pp. 435-444, May/June 2003

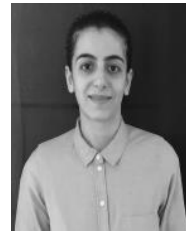

Shahideh Kiehbadroudinezhad received the B.S. degree in computer engineering from University of Shahid Bahonar of Kerman, Kerman, Iran, the M.S. degree in electronics science from Pune University, Pune, India, and the Ph.D. degree in communication engineering from Universitie Putra Malaysia, Serdang, Malaysia. From 2015 to 2017, she was an instructor and project adviser with the Institute of Computer Science, Andishesazanekahkeshan, Kong, Iran Since 2017, she has been a Researcher and Sessional Assistant Professor in the faculty of Engineering with the Electrical and Computer Engineering Department, Dalhousie University, Halifax, Canada. She is the author of two books, and several articles. Her research interests include the ML, algorithms, channel modeling and estimation, antenna propagation, optimization, and image/signal processing. Dr. Kieh holds one patent and was a recipient of the $1^{\text {st }}$ class honors degree in Electronics Science.

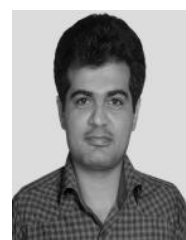

Adib Shahabia received the B.S. and M.S. degrees in electronics science from Pune University, Pune, India, and the second M.S. degree in electrical and computer engineering in 2019 from Dalhousie University, Halifax, Canada. His research interests include the communication, antenna propagation, FPGA, and image/signal processing. Mr. Shahabi holds one patent.

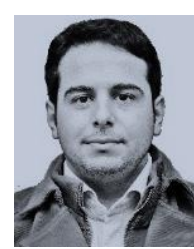

Mohammad Ali Kiehbadroudinezhad received the B.S degree in mechanic of machinery engineering from University of Uremia, Uremia, West Azerbaijan, Iran, the M.S. degree in mechanic of biosystem from University of Tehran, Tehran, Iran, and the Ph.D. candidate in mechanic of biosystem-renewable energy engineering from University of Tehran, Tehran, Iran. He was a researcher and project adviser in Institute of Education and Research, Amol, Iran. He is currently a Researcher and visiting student in the faculty of Engineering with the Electrical and Computer Engineering Department, Dalhousie University, Halifax, Canada. He is the author of six books, and several articles. His research interests include the Renewable system and energy, mathematic modeling and estimation, machine vision, and optimization. Mr. Kieh holds five patents. He was a recipient of the second place at the Elite Foundation of Iran's Inventions Festival in 2012. He was awarded the Bronze Medal of the International Invention Festival in 2014 